\title{
Prevalência de depressão em idosas participantes da Universidade para a Terceira Idade
}

\author{
Prevalence of depression in elderly women attending the University of Third Age
}

\author{
Tatiana Quarti Irigaray', Rodolfo Herberto Schneider ${ }^{2}$ \\ ${ }^{1}$ Psicóloga. Membro, Equipe Técnica, Universidade para a Terceira Idade - Universidade Federal do Rio Grande do Sul (UNITI/UFRGS), Porto \\ Alegre, RS. Mestranda em Gerontologia Biomédica, Pontifícia Universidade Católica do Rio Grande do Sul (PUCRS), Porto Alegre, RS. ${ }^{2}$ Médico \\ geriatra. Professor adjunto, Instituto de Geriatria e Gerontologia, PUCRS, Porto Alegre, RS.
}

\begin{abstract}
Resumo
Introdução: Estudos têm sugerido que o envelhecimento tem relação com a depressão. No entanto, os idosos podem apresentar ausência ou mesmo prevalência baixa de sintomatologia depressiva quando participam de atividades grupais de educação permanente.

Objetivos: Investigar a intensidade e prevalência de sintomatologia depressiva em idosas participantes da Universidade para a Terceira Idade (UNITI) da Universidade Federal do Rio Grande do Sul. Verificar se o tempo de participação na UNITI ajuda na melhora do humor e as características das participantes antes e após ingressarem na UNITI.

Método: Participaram 103 idosas integrantes da UNITI. Utilizou-se a Escala de Depressão Geriátrica (GDS) e uma ficha de dados sociodemográficos, que investigava o tempo de participação na UNITI e sua relação com sintomas depressivos, o papel da UNITI na melhora do humor e características das participantes antes e depois de entrarem na instituição. A amostra foi de conveniência.

Resultados: Observou-se ausência de sintomatologia depressiva na GDS na amostra estudada (média $=2,65$; desvio padrão $=2,42$ ). Do total de idosas, 80,6\% apresentaram escores normais na GDS, e nenhuma apresentou depressão grave. Observou-se diferença significativa entre depressão e tempo de participação na UNITI igual ou superior a 1 ano $(\mathrm{p} \leq 0,05)$. Verificou-se também que as participantes apresentaram melhoras no humor e nas áreas pessoal, intelectual, afetiva e social.

Conclusões: É possível envelhecer bem, com ausência de sintomatologia depressiva. Este estudo aponta para a existência de uma associação, a ser melhor investigada, entre menor intensidade de sintomatologia depressiva e tempo de participação na UNITI superior a 1 ano. Também sugere que a participação em uma universidade para a terceira idade traz melhoras aos idosos.

Descritores: Idoso, depressão, universidade para a terceira idade, envelhecimento.
\end{abstract}

\begin{abstract}
Background: Studies have suggested that aging is associated with depression. However, elderly can present absence or low prevalence of depressive symptoms when they participate in group activities of permanent education.

Objectives: To investigate the intensity and prevalence of depressive symptoms in elderly women attending the University of the Third Age (UNITI) at Universidade Federal do Rio Grande do Sul. To verify whether participation time in UNITI improves the mood and characteristics of participants before and after entering UNITI.

Method: One hundred and three UNITI students participated in the study. The Geriatric Depression Scale (GDS) and a form of sociodemographic data were used, investigating the participation time in UNITI and its relation with depressive symptoms, the role of UNITI in the improvement in the mood and characteristics of participants before and after entering this institution. A convenience sample was used. Results: Absence of depressive symptoms were observed in the GDS of the sample under investigation (mean $=2.65$; standard deviation = 2.42). Of all elderly women, $80.6 \%$ presented normal scores in the GDS and none presented severe depression. There was significant difference between depression and participation time in UNITI $\geq 1$ year ( $\mathrm{p} \leq 0.05$ ). Participants also presented improvement in mood and in personal, intellectual, affective and social areas.

Conclusions: It is possible to age well without depressive symptoms. This study shows the existence of an association, which needs further investigation, between minor intensity of depressive symptoms and participation time in UNITI > 1 year. It also suggests that attending a university of the third age brings improvements to the elderly people.
\end{abstract}

Keywords: Elderly, depression, university of the third age, aging. 


\section{Introdução}

A depressão é um dos problemas psiquiátricos mais comuns e importantes em idosos ${ }^{1}$. Ela caracteriza-se como um distúrbio da área afetiva ou do humor, que exerce forte impacto funcional em qualquer faixa etária. É um distúrbio de natureza multifatorial, que envolve inúmeros aspectos de ordem biológica, psicológica e social. É um estado de humor vivenciado por um grande número de indivíduos em algum momento de suas vidas, sendo muitas vezes relatada como tristeza, saudade, angústia, desânimo, entre outros ${ }^{2}$.

Batistoni ${ }^{3}$ define a depressão como uma síndrome psiquiátrica cujos principais sintomas são o humor deprimido e a perda de interesse ou prazer em quase todas as atividades. Em idosos, ela se manifesta de forma heterogênea, tanto em relação à sua etiologia quanto aos aspectos relacionados à sua apresentação e ao seu tratamento.

Segundo estudo de Beekman et al. ${ }^{4}$, há uma associação importante entre saúde física e depressão na velhice. Aspectos subjetivos e mais gerais de saúde física, tais como limitações funcionais e saúde percebida, estão mais fortemente associados com depressão do que a outras categorias de doenças.

A depressão pode ser responsável pela perda de autonomia e pelo agravamento de quadros patológicos preexistentes. É freqüentemente associada a maior risco de morbidades e de mortalidade, ocasionando o aumento na utilização dos serviços de saúde, à negligência no autocuidado, à adesão reduzida aos tratamentos terapêuticos e a maior risco de suicídio ${ }^{5}$. Em idosos, a presença de comorbidades e o uso de múltiplos fármacos fazem com que o diagnóstico e o tratamento da depressão se tornem mais complexos ${ }^{6}$.

Conforme estudo de Veras ${ }^{7}$, a prevalência de depressão em idosos na cidade do Rio de Janeiro variou de acordo com o grupo estudado - foi de 20,9\% no distrito com melhor nível socioeconômico e de 36,8\% no de pior nível socioeconômico. Para Frank \& Rodrigues $^{8}$, os transtornos depressivos entre idosos que vivem na comunidade variam de 4,8 a $14,6 \%$, e a prevalência em idosos hospitalizados ou institucionalizados é maior, atingindo $22 \%$. Por outro lado, os estudos que avaliam os sintomas clinicamente relevantes, mas que não preenchem critérios suficientes do Classificação Estatística Internacional de Doenças, volume 10 (CID-10) ou do Manual de Diagnóstico e Estatística das Perturbações Mentais, $4^{\mathrm{a}}$ edição (DSMIV), utilizando as escalas de sintomas, mostram uma prevalência na comunidade que varia de 6,4 a 59,3\% ${ }^{8}$.

A prevalência de depressão maior varia de 0,9 a $42 \%$ entre idosos caucasianos, e sintomas depressivos clinicamente relevantes variam de 7,2 a $49 \%$. Os principais fatores envolvidos nos distúrbios e sintomas depressivos são o gênero feminino, doenças somáticas, declínio cognitivo e funcional, falta ou perda de contato social e história de depressão anterior. Na velhice, os fatores de risco são falta ou perda de contatos sociais, história de depressão pregressa, viuvez, eventos de vida estressantes, institucionalização em casas asilares, renda baixa, insatisfação com o suporte social, isolamento social, ansiedade, falta de atividades sociais, nível educacional baixo e uso de medicação antidepressiva ${ }^{9}$.

Para Argimon \& Stein ${ }^{10}$, a depressão é um problema de saúde freqüente entre as pessoas de muita idade, embora a identificação seja muitas vezes difícil na prática clínica. Em um estudo realizado pelas autoras, os resultados levaram-nas à hipótese de que as pessoas mais idosas têm uma saúde física mais debilitada que outras com menos idade e, por isso, possuem maior risco de apresentarem sintomas depressivos, não em decorrência da idade mais avançada, mas por causa do estado de saúde mais precário.

O declínio da saúde física é freqüentemente pensado como o principal fator de relação para risco de depressão ${ }^{3,9}$, pois a presença de doenças muitas vezes leva os idosos a situações de incapacidade e maior dependência. Entretanto, desordens depressivas maiores são relativamente raras em idosos, enquanto sintomas depressivos que não têm critérios diagnósticos definidos pelo DSM-IV para depressão são comuns ${ }^{4}$. Por outro lado, estudos mostram um notável grau de estabilidade de sintomatologia depressiva em adultos com menos de 70 anos e um aumento em idades subseqüentes ${ }^{11,12}$. No decorrer do envelhecimento, é observado, principalmente, um aumento de sintomas somáticos e falta de bem-estar. Todavia, a quantidade de comorbidades é responsável por uma pequena parte dessa associação, devendo os sintomas somáticos ser vistos como sintomas da depressão e não como expressão de saúde física precária ${ }^{12}$.

A pobreza, a viuvez, a institucionalização e a solidão são identificadas também como fatores de risco para o desenvolvimento de sintomatologia depressiva. As mudanças nos papéis sociais, tais como a aposentadoria, freqüentemente ocasionam a diminuição da auto-estima, do ritmo das atividades e do interesse pelo dia-a-dia. A perda de pessoas próximas, como cônjuge, filhos ou amigos, também atua como fator desencadeante de sintomas depressivos ${ }^{2}$. A percepção do estado psicológico contribui como um fator adjuvante para o desencadeamento da depressão, pois os declínios reais ou imaginários nas capacidades, tal como demência e perda de memória, podem precipitar sintomatologia depressiva ${ }^{13}$.

Segundo alguns estudos, há uma grande variedade de doenças que têm relação etiológica direta com a depressão na velhice. As principais morbidades são o infarto do miocárdio, acidente vascular cerebral e doença 
de Parkinson ${ }^{14,15}$. Além disso, aspectos gerais da saúde física, tais como nível de prejuízo funcional e saúde percebida, são mais correlacionados com depressão do que diagnósticos específicos de doença ${ }^{16-18}$.

Na velhice, os eventos de vida estressantes e negativos são mais freqüentes e exigem do idoso a necessidade de adaptação, podendo contribuir para o aparecimento de depressão ${ }^{13}$. A depressão pode ser evitada, ou mesmo minimizada, através do apoio que o indivíduo recebe através das relações interpessoais ${ }^{19}$. Segundo Papalia et al. ${ }^{20}$, uma forte rede de amigos e de familiares pode ajudar os idosos a evitar ou enfrentar a depressão ${ }^{20}$. Dessa forma, determinados contextos afetivos podem tanto desencadear quanto proteger os indivíduos contra o surgimento de sintomatologia depressiva. O risco de depressão é aumentado na ausência de relacionamentos afetivos, de amizades e de vínculos de confiança, com cônjuge, parceiro ou amigo $^{19}$. Antonucci et al. ${ }^{21}$, avaliando diferenças entre homens e mulheres, relacionaram a qualidade das relações sociais, mais especificamente as relações sociais negativas, com manifestação de sintomatologia depressiva.

Um outro estudo encontrou relação direta entre engajamento social e menor prevalência de sintomas depressivos em idosos. Dessa forma, uma maior participação social atua como um importante modificador da saúde dos idosos, exercendo um papel fundamental na manutenção da saúde mental na velhice e surgindo como um meio de intervenção importante ${ }^{22}$. Para Murphy ${ }^{23}$, o suporte social tem um papel protetor na presença de um evento desagradável, funcionando como um redutor de impacto de eventos estressantes.

O presente estudo teve por objetivo investigar a intensidade e a prevalência de sintomatologia depressiva em idosas participantes da Universidade para a Terceira Idade (UNITI) da Universidade Federal do Rio Grande do Sul (UFRGS). Buscou-se analisar, também, se o tempo de participação na UNITI ajudava na melhora do humor e as características pessoais das participantes antes e após ingressarem no projeto.

A UNITI é um projeto de extensão, ensino e pesquisa que funciona junto ao Departamento de Psicologia Social e Institucional do Instituto de Psicologia da UFRGS. Iniciou suas atividades no primeiro semestre de 1991, estando, atualmente, no seu $16^{\circ}$ ano de funcionamento. O grupo da UNITI é composto semestralmente, em média por 150 pessoas da comunidade com 60 anos ou mais, em sua grande maioria mulheres idosas.

A dinâmica de funcionamento da UNITI ocorre em grande grupo, reunindo todos os participantes a cada segunda-feira, das $14 \mathrm{~h} 30 \mathrm{~min}$ às $17 \mathrm{~h}$, e em pequenos grupos, chamados subprojetos, que funcionam em outros dias da semana e em diferentes horários. As atividades ocorrem durante todo o ano letivo, no período de março a dezembro. No grande grupo, são desenvolvidos trabalhos que objetivam a conscientização sobre o processo de envelhecimento e abordados assuntos de interesses diversos, através de palestras, debates, seminários e trabalhos desenvolvidos pelas próprias participantes. Já os subprojetos trabalham com temas variados, de acordo com o interesse dos idosos. No segundo semestre de 2006, funcionaram os seguintes subprojetos: artesanato, cine-debate, cultura, cultura espanhola, estudos e debates, expressão corporal, grupo social, literatura, meio ambiente e unimúsica. Os subprojetos são coordenados pelos próprios participantes da UNITI, podendo ser criados novos grupos à medida que entram novos integrantes dispostos a desenvolver algum estudo ou atividade. Eles são autogerenciados e funcionam sob a supervisão da equipe técnica, que conta com uma psicóloga e gerontóloga, duas psicólogas, uma pedagoga, um técnico em informática e alunos do curso de graduação em psicologia.

Segundo Castro ${ }^{24}$, o trabalho da UNITI fundamenta-se nos princípios da educação permanente, que pode ser definida como uma proposta pedagógica participativa, que oportuniza ao idoso experiências relativas ao seu tempo e à construção de seu espaço. $\mathrm{O}$ objetivo principal da UNITI é potencializar os recursos humanos constituídos pela faixa etária dos 60 anos ou mais. Utiliza-se de estratégias que viabilizem mudanças sociais que permitam ao idoso o direito de participar de acordo com seus próprios recursos. A dinâmica de trabalho se apóia na própria capacidade dos participantes idosos e nas suas necessidades específicas, dentro de uma filosofia que privilegia a autodescoberta. O projeto UNITI proporciona aos idosos uma revisão no processo de socialização, permitindo e oferecendo experiências de aprendizagens sobre o ritmo e a seqüência das mudanças evolutivas do curso de vida.

\section{Método}

\section{Amostra}

A amostra foi composta por 103 idosas, de um total de 155 participantes da UNITI, sendo 153 mulheres e dois homens. As idosas tinham idade entre 60 e 86 anos e escolaridade mínima de 7 anos de estudo, ou seja, até a $7^{\mathrm{a}}$ série do ensino fundamental. O método amostral utilizado foi o de conveniência. Todas as 153 integrantes da UNITI foram convidadas a participar do estudo, mas apenas 109 consentiram em participar. Foram excluídas seis pessoas da amostra por apresentarem menos de 60 anos. Nenhuma idosa foi excluída por apresentar sintomas sugestivos de demência. 


\section{Instrumentos}

\section{Ficha de dados sociodemográficos}

A ficha de dados sociodemográficos incluiu as seguintes variáveis: sexo, idade, estado civil, escolaridade, renda, situação de moradia, ano de aposentadoria, ocupação, ano de ingresso na UNITI, número de filhos, netos e bisnetos, atividade física realizada, percepção de saúde, uso de medicação, realização de trabalho voluntário e uso de cigarro e bebida (quantidade e freqüência). Também avaliou as seguintes questões: 1) Ano e semestre de ingresso na UNITI; 2) “A UNITI auxilia na melhora do seu humor?” - Opções de resposta categorizadas em: “nada”, “muito pouco”, “médio", "muito” e “completamente”; 3) "Liste três características suas antes de entrar para a UNITI e outras três que surgiram depois que entrou para a UNITI”.

\section{Escala de Depressão Geriátrica}

A Escala de Depressão Geriátrica (GDS) ${ }^{25}$ é uma medida utilizada para o rastreamento de depressão em idosos, sendo um dos instrumentos mais freqüentes utilizados para esse fim. É composta por 15 itens na versão curta e foi desenvolvida especialmente para o rastreamento dos transtornos de humor em idosos, com perguntas que evitam a esfera das queixas somáticas. São perguntas fechadas, apresentando respostas do tipo “sim” ou “não”. Diversos estudos mostraram que a GDS oferece medidas válidas e confiáveis ${ }^{26,27}$.

\section{Procedimentos para coleta de dados}

Inicialmente, foram realizados contatos com a UNITI e, após, procedeu-se com a inclusão das participantes na amostra. Todas as idosas preencheram o termo de consentimento livre e esclarecido e, a partir daí, foram convidadas a responder acerca de informações sociodemográficas e sintomatologia de depressão (GDS) ${ }^{25}$.

Tanto a ficha de dados sociodemográficos quanto a GDS são de caráter auto-administrável; contudo, um auxiliar de pesquisa (estudante de psicologia) sempre esteve à disposição das idosas para qualquer esclarecimento. A aplicação dos instrumentos foi feita em grupos de até 15 participantes.

O projeto foi aprovado pelo Comitê de Ética em Pesquisa da Pontifícia Universidade Católica do Rio Grande do Sul, Porto Alegre (RS), Brasil (protocolo $n^{\circ}$ 06/0309).

\section{Procedimentos para análise dos dados}

A descrição das variáveis foi realizada através das freqüências absolutas e relativas, bem como média (M) e desvio padrão (DP), sendo que a GDS foi agrupada por tercis.
Para a comparação dos valores da variável depressão e tempo de participação na UNITI, foi utilizado o teste $t$ de Student.

Foram consideradas significativas as associações com valores de $\mathrm{p} \leq 0,05$.

Para análise dos dados, foi utilizado o programa SPSS para Windows, versão 13.0.

\section{Resultados}

A Tabela 1 mostra as características sociodemográficas da amostra em estudo. Conforme pode ser observado, a amostra contou com 103 mulheres idosas na faixa etária entre 60 e 86 anos (idade média de 69,2 anos; DP =6,46). O estado civil mais freqüente entre as idosas foi o de viúva (44,7\%); a escolaridade mais prevalente foi o ensino superior (40,8\%); e a renda de seis a 10 salários mínimos foi a mais freqüente (38,8\%). Referente à situação de moradia, prevaleceu a condição morando com a família (52,4\%); a profissão de professora foi a ocupação anterior mais freqüente (28,1\%); e a aposentadoria foi a ocupação atual prevalente (73,8\%). A percepção de saúde predominante foi a de saudável (81,6\%); o uso de medicação $(89,3 \%)$ e a realização de atividade física (74,8\%) também foram citados entre as idosas. A maior parte das idosas (84,4\%) participa da UNITI há mais de 1 ano.

A Tabela 2 apresenta a intensidade de depressão nas idosas conforme dados da GDS total $(\mathrm{M}=2,65$; $\mathrm{DP}=$ 2,42 ) e também conforme agrupamento por tercis. As idosas responderam a uma pontuação mínima de zero e máxima de 10 na GDS. Segundo a GDS, agrupada em tercis, o primeiro tercil $(<5)$, que corresponde ao grupo com escores normais, foi a pontuação mais freqüente (80,6\%). Na avaliação do segundo tercil (5 a 10), que corresponde ao grupo com presença de depressão leve a moderada, a freqüência de respostas foi de 19,4\%. Já na avaliação do terceiro tercil, que corresponde ao grupo com maior intensidade de sintomas depressivos e depressão grave, não ocorreram, em nenhum caso, escores suficientes para pontuar esse tipo de depressão.

A correlação entre a variável depressão (GDS) e o tempo de participação na UNITI também foi avaliada. Através do teste $t$ de Student, foi possível verificar uma diferença significativa ( $\mathrm{p} \leq 0,05$ ) para as variáveis depressão (GDS) e tempo de participação na UNITI menor ou igual versus superior a 1 ano. Conforme essa comparação, as pessoas que participavam há mais de 1 ano apresentaram menos sintomas de depressão (GDS $=2,39$ ) em relação àquelas que participavam há 1 ano ou menos (GDS = 4,06).

As respostas dadas sobre a importância da participação na UNITI na melhora do humor são apresentadas na Tabela 3. 
Tabela 1 - Características sociodemográficas da amostra em estudo $(n=103)$

\begin{tabular}{|c|c|c|}
\hline Variáveis & $\mathbf{n}$ & $\%$ \\
\hline \multicolumn{3}{|l|}{ Sexo } \\
\hline Feminino & 103 & 100 \\
\hline Idade, média (desvio padrão) & $69,2(6,46)$ & \\
\hline \multicolumn{3}{|l|}{ Estado civil } \\
\hline Solteira & 5 & 4,9 \\
\hline Casada/com companheiro & 32 & 31,0 \\
\hline Viúva & 46 & 44,7 \\
\hline $\begin{array}{l}\text { Separada/divorciada/sem } \\
\text { companheiro }\end{array}$ & 20 & 19,4 \\
\hline \multicolumn{3}{|l|}{ Escolaridade } \\
\hline Ensino fundamental & 21 & 20,4 \\
\hline Ensino médio & 40 & 38,8 \\
\hline Ensino superior & 42 & 40,8 \\
\hline \multicolumn{3}{|l|}{ Renda } \\
\hline 1 a 5 salários mínimos & 22 & 21,3 \\
\hline 6 a 10 salários mínimos & 40 & 38,8 \\
\hline 11 a 15 salários mínimos & 22 & 21,4 \\
\hline 16 a 20 salários mínimos & 7 & 6,8 \\
\hline Mais de 20 salários mínimos & 12 & 11,7 \\
\hline \multicolumn{3}{|l|}{ Situação de moradia } \\
\hline Morando sozinha & 49 & 47,6 \\
\hline Morando com a família & 54 & 52,4 \\
\hline \multicolumn{3}{|l|}{ Ocupação anterior } \\
\hline Professora & 29 & 28,1 \\
\hline Funcionária pública & 21 & 20,4 \\
\hline Do lar & 15 & 14,6 \\
\hline Comerciante & 7 & 6,8 \\
\hline Secretária & 7 & 6,8 \\
\hline Auxiliar de enfermagem & 3 & 2,9 \\
\hline Outras & 21 & 20,4 \\
\hline \multicolumn{3}{|l|}{ Ocupação atual } \\
\hline Aposentada & 76 & 73,8 \\
\hline Do lar & 20 & 19,4 \\
\hline Outra & 7 & 6,8 \\
\hline \multicolumn{3}{|l|}{ Percepção de saúde } \\
\hline Saudável & 84 & 81,6 \\
\hline Doente & 2 & 1,9 \\
\hline Nem saudável, nem doente & 17 & 16,5 \\
\hline \multicolumn{3}{|l|}{ Uso de medicação } \\
\hline Sim & 92 & 89,3 \\
\hline Não & 11 & 10,7 \\
\hline \multicolumn{3}{|l|}{ Realiza atividade física } \\
\hline Sim & 77 & 74,8 \\
\hline Não & 26 & 25,2 \\
\hline \multicolumn{3}{|l|}{ Tempo de participação na UNITI } \\
\hline Mais de 1 ano & 87 & 84,4 \\
\hline Menos de 1 ano & 16 & 14,6 \\
\hline
\end{tabular}

UNITI = Universidade para a Terceira Idade.

As características das idosas antes e após ingressarem na UNITI são mostradas na Tabela 4. As participantes salientaram aspectos pessoais e sentimentos vivenciados.
Tabela 2 - Média, desvio padrão e freqüência da Escala de Depressão Geriátrica $(n=103)$

\begin{tabular}{lc}
\hline Variáveis & n (\%) \\
\hline GDS total, média (desvio padrão) & $2,65(2,42)$ \\
GDS $1^{\circ}$ tercil $(<5)$ & $83(80,6)$ \\
GDS $2^{\circ}$ tercil $(5-10)$ & $20(19,4)$ \\
GDS 3 & tercil $(11-15)$ \\
\hline
\end{tabular}

GDS = Escala de Depressão Geriátrica.

Tabela 3 - Freqüência de respostas sobre a melhora do humor com a participação na UNITI $(n=103)$

\begin{tabular}{lc}
\hline $\begin{array}{l}\text { Resposta à pergunta: “A UNITI ajuda na } \\
\text { melhora do seu humor?” }\end{array}$ & n (\%) \\
\hline Não respondeu & $1(1,0)$ \\
Nada & $2(1,9)$ \\
Muito pouco & $6(5,8)$ \\
Médio & $9(8,7)$ \\
Muito & $69(67,0)$ \\
Completamente & $16(15,6)$ \\
\hline UNITI = Universidade para a Terceira Idade.
\end{tabular}

\section{Discussão}

Diferentes estudos têm sugerido que o envelhecimento apresenta uma estreita relação com a presença de depressão. No entanto, o nosso estudo mostrou uma ausência de sintomatologia depressiva na GDS ( $M=2,65$; DP = 2,42) na amostra estudada. Esse resultado é corroborado por vários outros estudos ${ }^{28-30}$.

Uma associação entre menor intensidade de depressão e participação em uma universidade da terceira idade foi encontrada por Loures \& Gomes ${ }^{28}$. Para as autoras, a hipótese da associação direta entre intensidade de depressão e participação em um curso da Universidade Aberta à Terceira Idade da Universidade Católica de Goiás (UNATI/UCG) foi reforçada ao constatarem a diminuição da prevalência de depressão ao término do curso, pois a freqüência ao curso provavelmente fez com que os alunos se sentissem menos abandonados, com menos solidão e menos desprezados, pelo convívio entre eles próprios, com seus colegas e/ou com seus professores ${ }^{28}$. Outros estudos reforçam a hipótese de melhoras nas áreas pessoal, intelectual, afetiva e social com a participação em universidades da terceira idade ${ }^{29,30}$. Cachioni ${ }^{29}$ relatou que a participação das alunas na Universidade da Terceira Idade de São Francisco levou-as a se sentirem mais valorizadas socialmente e mais respeitadas. Além disso, as participantes obtiveram ganhos educacionais, 
Tabela 4 - Freqüência de respostas sobre

características pessoais antes e após ingressar na UNITI

\begin{tabular}{lc}
\hline Período (antes/depois de ingressar na UNITI) & n (\%) \\
\hline Antes de ingressar na UNITI (n=285) & $50(17,5)$ \\
Não tinham objetivos de vida & $41(14,4)$ \\
Sentiam-se solitárias & $40(14,0)$ \\
Tinham acesso a pouca informação, conhecimentos e cultura em geral & $38(13,3)$ \\
Sentiam tristeza, ansiedade e pessimismo & $31(10,9)$ \\
Sentiam-se inibidas e apresentavam timidez & $28(9,8)$ \\
Tinham baixa auto-estima & $25(8,8)$ \\
Tinham tempo ocioso, eram desocupadas & $12(4,2)$ \\
Liam pouco & $10(3,5)$ \\
Faziam sempre as mesmas coisas, mesma rotina & $6(2,1)$ \\
Tinham conceitos errados sobre a velhice & $4(1,4)$ \\
Apresentavam preocupação com outras coisas & \\
Após ingressar na UNITI (n = 368) & $63(17,1)$ \\
Não se sentem mais solitárias & $60(16,3)$ \\
Sentem-se mais alegres e felizes & $45(12,2)$ \\
Conquistaram um novo sentido de vida & $41(11,1)$ \\
Apresentam melhor auto-estima & $39(10,6)$ \\
Adquiriram mais conhecimentos, informações e cultura & $37(10,1)$ \\
Têm novas atividades & $30(8,2)$ \\
Sentem-se menos inibidas e tímidas & $17(4,6)$ \\
Apresentam mais cuidados com a saúde & $15(4,1)$ \\
Lêem mais & $13(3,5)$ \\
Aceitam melhor a idade & $8(2,2)$ \\
Sentem-se mais calmas e tranqüilas &
\end{tabular}

UNITI = Universidade para a Terceira Idade.

que se refletiram em maior autoconfiança e autoeficácia, em termos cognitivos e de produtividade. Na pesquisa realizada por Erbolato $^{30}$ na Universidade da Terceira Idade da Pontifícia Universidade Católica de Campinas, os alunos revelaram que a participação no programa trouxe benefícios nas áreas pessoal, intelectual e social. Também foram relatadas mudanças positivas em suas concepções de envelhecimento, nos cuidados com a saúde, na rotina de vida, no enfrentamento de problemas, no relacionamento com os amigos e na autopercepção. De forma diferente, o estudo de Leite et al. ${ }^{31}$ encontrou um percentual importante de depressão $(24,02 \%)$ nos idosos que participaram do programa Universidade Aberta à Terceira Idade, da Universidade Federal de Pernambuco, cuja maioria foi classificada em depressão menor. Os casos de depressão apresentaram relevante relação com as variáveis preocupação, dor de cabeça, pouca disposição, irritação, tristeza e insatisfação.

No nosso estudo, uma possível explicação para a ausência de sintomatologia depressiva poderia ser devido à influência do trabalho realizado na UNITI, que tem como objetivo principal a valorização do indivíduo idoso, enquanto ser pensante ativo e participante da sociedade. Na UNITI, o grupo de idosos busca e decide sobre as motivações da própria vida, repensa e reformula a própria história, liberando e usando, produtivamente, os recursos de sua personalidade $^{32}$.

As idosas, antes de entrarem na UNITI, não tinham objetivos de vida, sentiam-se solitárias, tinham acesso a pouca informação e cultura em geral e sentiam tristeza. Uma hipótese de explicação para a vivência desses sentimentos poderia estar nas características da nossa amostra, pois somente $31 \%$ têm companheiro, quase metade mora sozinha, e a maioria é aposentada. De acordo com Papalia et al. ${ }^{20}$, as mulheres, por viverem mais tempo do que os homens e por terem maior probabilidade de ficarem viúvas, têm duas vezes mais chances do que os homens de viverem sozinhas, aumentando essa probabilidade com a idade. Entre os idosos de 85 anos ou mais, quase $60 \%$ das mulheres e $30 \%$ dos homens vivem sozinhos. O fato de viverem sozinhas não determina a vivência da solidão na velhice. Outros fatores podem desempenhar um papel mais significativo na vulnerabilidade à solidão, como personalidade, habilidade cognitivas, saúde física e uma rede social ampla. Além disso, a aposentadoria aparece 
como um marcador importante do envelhecimento dessas idosas, que pode ter ocasionado o corte de vínculos, o empobrecimento relacional e a perda da identidade.

Os sentimentos gerados nos idosos pelo processo de envelhecimento são vividos, na maioria das vezes, como algo novo e desestabilizador, resultando em ansiedade, medo, conflito, insegurança e, principalmente, sentimentos de solidão. Segundo Castro $^{32}$, a solidão teria origem na convicção de que não há "grupo" a que se pertença, ou seja, ocorre a perda da identidade em relação a si próprio. Dessa forma, a inserção do idoso no grupo da UNITI dar-se-ia através do sentimento de pertencimento, que seria uma forma de vinculação e reconstrução da própria identidade enquanto sujeito idoso ${ }^{32}$.

Através da participação na UNITI, os idosos têm a possibilidade de interação com outras pessoas, refazendo um novo círculo de amizades. Também adquirem novos conhecimentos e se atualizam, o que se reflete em maior abertura para conversar e se relacionar com os familiares e outras pessoas. Em decorrência desses e de outros fatores vivenciados no grupo, os idosos apresentam uma melhor auto-estima, que se reflete num senso de maior auto-eficácia. De uma forma simples, auto-estima significa gostar de si mesmo, isto é, apreciar-se de modo genuíno e realista. A auto-estima está diretamente relacionada com a resolução dos problemas (auto-eficácia) com os quais a pessoa se defronta ${ }^{33}$. A auto-eficácia é a própria crença da pessoa em sua capacidade para organizar, resolver e executar as ações requeridas para alcançar determinados resultados. É um mecanismo fundamental, porque prediz o nível de realização que as pessoas atingem e tem forte impacto no pensamento, no afeto, na motivação e na ação ${ }^{34}$. Assim, o idoso que tem uma boa auto-estima sente-se mais capaz para resolver os problemas, e quanto mais capaz se percebe, maior é sua auto-estima.

Nas universidades para a terceira idade, geralmente os idosos são incentivados a conquistar ou manter um estilo de vida o mais saudável possível dentro da realidade biopsicossocial de cada um. Nesses espaços, a manutenção da máxima capacidade funcional e a conquista e a preservação da autonomia, de uma boa qualidade de vida e da independência são incentivadas. Os idosos têm a possibilidade de adquirir novas informações, ampliar conhecimentos e, principalmente, conquistar novas amizades, afastando a solidão e, assim, a depressão.

No Brasil, a partir da década de 1990, ocorreu a multiplicação dos programas voltados para adultos maduros e idosos nas universidades brasileiras. Entre 1990 e 1999, esses programas cresceram de seis para cerca de 140. Atualmente, essas instituições se espalham por todo o país, existindo cerca de 200 programas em instituições públicas e privadas ${ }^{35}$. Segundo Debert ${ }^{36}$, os programas foram criados em razão da visibilidade alcançada pela velhice na década de 1990 , pois a preocupação com o envelhecimento e com a melhoria da qualidade de vida dos idosos é algo recente. Esses espaços estão envolvidos na luta contra os preconceitos e os estereótipos atribuídos à velhice no contexto brasileiro, buscando demonstrar que é possível atribuir novos conteúdos à velhice ${ }^{36}$. Dentro dessa perspectiva, nos próximos anos, a velhice não será mais um fato novo; talvez os preconceitos, estereótipos e discriminações em relação aos idosos já estejam flexibilizados. Assim, o futuro da velhice e das universidades da terceira idade poderá ser influenciado pelo modo como os indivíduos serão educados em relação ao seu destino e papel na sociedade em que vivem.

Outro fator que merece destaque para a ausência de sintomatologia depressiva são as características sociodemográficas da amostra estudada. A nossa amostra foi composta, em sua maioria, por idosas com nível de escolaridade elevado. Essa característica poderia explicar o baixo escore total na GDS. No estudo de Trentini et al. ${ }^{37}$, a escolaridade foi a variável mais consistentemente associada com a intensidade de depressão. Essa associação dar-se-ia de forma inversa: quanto maior a escolaridade, menor a intensidade de sintomas psicológicos ou de somatizações. Dessa forma, a escolaridade exerceria um papel protetor para sintomas depressivos ou para sua expressão. Outros estudos também têm demonstrado uma relação entre menor escolaridade e maior número de idosos deprimidos ${ }^{31,38}$.

Dentre as características da amostra estudada, convém destacar que a mesma foi composta, em sua maioria, por mulheres de classe média, aposentadas, com nível de escolaridade elevado, sendo a profissão de professora a ocupação anterior mais freqüentemente citada. Uma possível explicação para esse achado talvez seja o fato de que a UNITI é um projeto que funciona dentro da universidade. Como conseqüência, atrai pessoas com boa saúde física e níveis financeiro e educacional elevados. Idosos de menor escolaridade podem ter desempenhado trabalhos fisicamente mais desgastantes, ter tido menos acesso à saúde e, por isso, podem ter mais dificuldades em ir até uma universidade e participar de um programa como a UNITI. De acordo com Silva ${ }^{39}$, as pessoas que ao longo de suas vidas tiveram acesso às oportunidades educacionais são melhores candidatas a encarar a educação como um meio de enfrentar os desafios ocasionados pelo processo de envelhecimento. Desta forma, a educação parece ser um forte indicador de enfrentamento bem-sucedido da velhice (indivíduos que tiveram experiências 
educacionais ao longo da vida teriam uma vantagem adicional na vivência da velhice).

Embora a característica atual do envelhecimento mostre uma maior predominância de mulheres idosas, é importante ressaltar, ainda, que o estudo na UNITI foi conduzido com uma amostra relativamente restrita, formada somente por mulheres idosas, o que pode não refletir a realidade dos idosos em geral. $\mathrm{O}$ fato de a amostra ter sido composta somente por mulheres devese à participação quase inexistente de homens idosos em programas para a terceira idade. Na UNITI, o número de homens idosos participantes nunca ultrapassou $5 \%$ da totalidade ao longo dos seus 16 anos de funcionamento. Segundo Debert ${ }^{36}$, uma característica marcante dos programas para a terceira idade é a predominância de mulheres, que representam a maioria da população total. Os homens idosos geralmente fazem parte de associações, federações e confederações de aposentados ou sindicatos de classes profissionais. Segundo a autora, esse fato pode estar relacionado às diferenças entre homens e mulheres quanto à representação da velhice e a como esses indivíduos percebem as mudanças ocorridas no envelhecimento - ou seja, as mulheres com mais interesse cultural e os homens mais voltados para a política. Assim, os papéis desempenhados pelos gêneros estariam direcionados em diferentes caminhos, estando os homens mais voltados aos direitos do cidadão e redistribuição da riqueza, e as mulheres, às mudanças culturais amplas.

No presente estudo, outra associação importante encontrada foi entre o tempo de participação na UNITI (maior de 1 ano) e menor intensidade de sintomatologia depressiva. Ele aponta a existência dessa correlação, que merece uma investigação mais aprofundada; porém, estudos que explicam essa associação ainda são incipientes.

Os dados desta pesquisa não podem ser generalizados para toda a população. Eles são indicativos de que é possível envelhecer bem e de que os programas para a terceira idade podem auxiliar os idosos na reconstrução de novos planos de vida, no combate à solidão e no desenvolvimento de novas habilidades. Este estudo contribui para o fortalecimento do argumento de que a educação ao longo de toda a vida é um poderoso preditor de um envelhecimento feliz e com qualidade.

\section{Conclusões}

A depressão não é necessariamente uma morbidade integrante da velhice. A média geral baixa na GDS das idosas sugere ausência de depressão. Dessa forma, o presente estudo mostra que é possível envelhecer bem, com ausência de sintomatologia depressiva. O uso da GDS apontou para uma associação entre menor intensidade de sintomatologia depressiva e tempo de participação na UNITI superior a 1 ano. Assim, quanto mais tempo de participação, menor a intensidade de sintomas depressivos.

A escolaridade mais elevada parece exercer influência na prevalência de depressão; assim, estudos complementares voltados para a implementação de políticas educacionais voltadas à população idosa podem colaborar na eficácia da saúde física e psicológica dos idosos, atuando, secundariamente, como fatores protetores contra a depressão na velhice.

\section{Referências}

1. Carson A, Margolin R. Depression in older patients with neurologic illness: Causes, recognition, management. Cleve Clin J Med. 2005;72 Suppl 3:S52-64.

2. Gordilho A. Depressão, ansiedade outros distúrbios afetivos e suicídio. In: Freitas EV, Py L, Neri AL, Cançado FAX, Gorzoni ML, Rocha SM, orgs. Tratado de geriatria e gerontologia. Rio de Janeiro: Koogan; 2002. p. 204-15.

3. Batistoni SST. Depressão. In: Neri AL. Palavras-chave em gerontologia. Campinas, SP: Alínea; 2005. p. 59-61.

4. Beekman AT, Penninx BW, Deeg DJ, Ormel J, Braam AW, van Tilburg W. Depression and physical health in later life: results from the Longitudinal aging study Amsterdam (LASA). J Affect Disord. 1997;46:219-31.

5. Katona C, Livingston G, Manela M, Leek C, Mullan E, Orrell M et al. The symptomatology of depression in the elderly. Int Clin Psychopharmacol. 1997;12 Suppl 7:S19-23.

6. Paradela EMP, Lourenço RA, Veras RP. Validação da escala de depressão geriátrica em um ambulatório geral. Rev Saúde Pública. 2005;39(6):918-23.

7. Veras RP, Coutinho ESF. Estudo de prevalência de depressão e síndrome cerebral orgânica na população de idosos, Brasil. Rev Saúde Pública. 1991;25:209-17.

8. Frank MH, Rodrigues NL. Depressão, ansiedade, outros distúrbios afetivos e suicídio. In: Freitas EV, Py L, Cançado FAX, Doll, J, Gorzoni, ML, orgs. Tratado de geriatria e gerontologia. Rio de Janeiro: Koogan; 2006. p. 376-86.

9. Djernes JK. Prevalence and predictors of depression in populations of elderly: a review. Acta Psychiatr Scand. 2006;113:372-87.

10. Argimon IIL, Stein LM. Habilidades cognitivas em indivíduos muito idosos: um estudo longitudinal. Cad Saúde Pública. 2005;21(1):6472.

11. Rothermund K, Brandtstadter J. Depression in later life: crosssequential patterns and possible determinants. Psychol Aging. 2003;18:80-90.

12. Nguyen HT, Zonderman AB. Relationship between age and aspects of depression: consistency and reliability across two longitudinal studies. Psychol Aging. 2006;21:119-26.

13. Stuart-Hamilton I. A psicologia do envelhecimento: uma introdução. Porto Alegre: Artmed; 2002.

14. Eastwood MR, Rifat SL, Nobbs H, Ruderman J. Mood disorder following cerebrovascular accident. Br J Psychiatry. 1989;154:195200.

15. Cummings JL. Depression and Parkinson's disease: a review. Am J Psychiatry. 1992;149:443-54.

16. Kinzie JD, Lewinsohn P, Maricle R, Teri L. The relationship of depression to medical illness in an older community population. Compr Psychiatry. 1986;27:241-6.

17. Kennedy GJ, Kelman HR, Wisniewski W, Metz H, Bijur P. Hierarchy of characteristics associated with depressive symptoms in an urban elderly sample. Am J Psychiatry. 1989;146: 220-5. 
18. Beekman AT, Kriegsman DM, Deeg DJ, van Tilburg W. The association of physical and depressive symptoms in the older population: age and sex differences. Soc Psychiatr Epidemiol. 1995;30:32-8.

19. Jefferson JW, Greist JH. Depression and antidepressants: a guide. Midedleton: Dean Foundation; 1997.

20. Papalia DE, Olds SW, Feldman RD. Desenvolvimento humano. Porto Alegre: Artmed; 2006.

21. Antonucci TC, Lansford JE, Akiyama H. Differences between men and women in social relations, resource deficits, and depressive symptomatology during later life in four nations. J Soc Issues. 2002;58(4):767-83.

22. Glass TA, De Leon CFM, Bassuk, SS, Berkman LF. Social engagement and depressive symptoms in late life: longitudinal findings. J Aging Health. 2006;18:604-28.

23. Murphy E. Social origins of depression in old age. Br J Psychiatry. 1982;141:135-42.

24. Castro, OP. Vivendo em seu corpo: uma questão de consciência e de criatividade. In: Castro OP, org. Envelhecer: revisitando o corpo. Sapucaia do Sul: Notadez; 2004. p. 13-30.

25. Sheikh JI, Yesavage JA. Geriatric depression scale (GDS): recent evidence and development of a shorter version. Clin Gerontol. 1986;5: 165-73.

26. Almeida OP, Almeida SA. Confiabilidade da versão brasileira da escala de depressão em geriatria (GDS) versão reduzida. Arq Neuropsiquiatr. 1999;57:421-6.

27. Almeida OP, Almeida SA. Short versions of the geriatric depression scale: a study of their validity for the diagnosis of a major depressive episode according to ICD-10 na DSM-IV. Int J Geriatr Psychiatry. 1999;14:858-65.

28. Loures MC, Gomes L. Prevalência da depressão entre os alunos da universidade aberta à terceira idade, Universidade Católica de Goiás no início e no término de seu curso. In: Faleiros VP, Loureiro, AML, orgs. Desafios do envelhecimento: vez, sentido e voz. Brasília: Universa; 2006. p. 139-58.
29. Cachioni M. Envelhecimento bem-sucedido e a participação numa universidade para a terceira idade: a experiência dos alunos da Universidade São Francisco [dissertação]. Campinas: Universidade Estadual de Campinas; 1998.

30. Erbolato RMPL. Universidade da terceira idade: Avaliação e perspectivas de alunos e ex-alunos [dissertação]. Campinas: Pontifícia Universidade Católica de Campinas; 1996.

31. Leite VMM, Carvalho EMFC, Barreto KML, Falcão IV. Depressão e envelhecimento: estudo nos participantes do Programa Universidade Aberta à Terceira Idade. Rev Bras Saúde Matern Infant. 2006;6(1):31-8.

32. Castro, OP. O processo grupal, a subjetividade e a ressignificação da velhice. In: Castro OP, org. Velhice que idade é esta? Uma construção psicossocial do envelhecimento. Porto Alegre: Síntese; 1998. p. 3555 .

33. Erbolato RMPL. Gostando de si mesmo: a auto-estima. In: Neri Al, Freire AS, orgs. E por falar em boa velhice. Campinas, SP: Papirus; 200. p. 33-54.

34. Bandura A. Self-efficacy: the exercise of control. New York: W. H. Freeman; 1997.

35. Cachioni M. Universidade da Terceira Idade. In: Neri AL. Palavraschave em gerontologia. Campinas: Alínea; 2005. p. 207-10.

36. Debert GG. A reinvenção da velhice: socialização e processos de reprivatização do envelhecimento. São Paulo: EDUSP; 1999.

37. Trentini CM, Xavier FMF, Chachamovich E, Rocha NS, Hirakata VN, Fleck, MPA. A influência dos sintomas somáticos no desempenho dos idosos no Inventário de Depressão de Beck (BDI). Rev Bras Psiquiatr. 2005;27(2):119-23.

38. Cacciatore F, Gallo C, Ferrara N, Abete P, Paolisso G, Canonico S, et al. Morbidity patterns in aged population in southern Italy: a survey sampling. Arch Gerontol Geriatr. 1998;26: 201-13.

39. Silva FP. Crenças em relação à velhice, bem-estar subjetivo e motivos para freqüentar universidade da terceira idade [dissertação]. Campinas: Universidade Estadual de Campinas; 1999. 